Edition KWV 
Die „Edition KWV“ beinhaltet hochwertige Werke aus dem Bereich der Wirtschaftswissenschaften. Alle Werke in der Reihe erschienen ursprünglich im Kölner Wissenschaftsverlag, dessen Programm Springer Gabler 2018 übernommen hat.

Weitere Bände in der Reihe http://www.springer.com/series/16033 
Timmy Wengerofsky

\section{Die materielle Stetigkeit im Handels- und Steuerbilanzrecht}

黛 Springer Gabler 
Timmy Wengerofsky

Wiesbaden, Deutschland

Bis 2018 erschien der Titel im Kölner Wissenschaftsverlag, Köln

Dissertation, Universität zu Köln, 2014

\section{Edition KWV}

ISBN 978-3-658-24654-9 ISBN 978-3-658-24655-6 (eBook)

https://doi.org/10.1007/978-3-658-24655-6

Die Deutsche Nationalbibliothek verzeichnet diese Publikation in der Deutschen Nationalbibliografie; detaillierte bibliografische Daten sind im Internet über http://dnb.d-nb.de abrufbar.

\section{Springer Gabler}

(c) Springer Fachmedien Wiesbaden GmbH, ein Teil von Springer Nature 2014, Nachdruck 2019

Ursprünglich erschienen bei Kölner Wissenschaftsverlag, Köln, 2014

Das Werk einschließlich aller seiner Teile ist urheberrechtlich geschützt. Jede Verwertung, die nicht ausdrücklich vom Urheberrechtsgesetz zugelassen ist, bedarf der vorherigen Zustimmung des Verlags. Das gilt insbesondere für Vervielfältigungen, Bearbeitungen, Übersetzungen, Mikroverfilmungen und die Einspeicherung und Verarbeitung in elektronischen Systemen.

Die Wiedergabe von allgemein beschreibenden Bezeichnungen, Marken, Unternehmensnamen etc. in diesem Werk bedeutet nicht, dass diese frei durch jedermann benutzt werden dürfen. Die Berechtigung zur Benutzung unterliegt, auch ohne gesonderten Hinweis hierzu, den Regeln des Markenrechts. Die Rechte des jeweiligen Zeicheninhabers sind zu beachten.

Der Verlag, die Autoren und die Herausgeber gehen davon aus, dass die Angaben und Informationen in diesem Werk zum Zeitpunkt der Veröffentlichung vollständig und korrekt sind. Weder der Verlag, noch die Autoren oder die Herausgeber übernehmen, ausdrücklich oder implizit, Gewähr für den Inhalt des Werkes, etwaige Fehler oder Äußerungen. Der Verlag bleibt im Hinblick auf geografische Zuordnungen und Gebietsbezeichnungen in veröffentlichten Karten und Institutionsadressen neutral.

Springer Gabler ist ein Imprint der eingetragenen Gesellschaft Springer Fachmedien Wiesbaden GmbH und ist ein Teil von Springer Nature

Die Anschrift der Gesellschaft ist: Abraham-Lincoln-Str. 46, 65189 Wiesbaden, Germany 


\section{Geleitwort}

Mit dem Bilanzrechtsmodernisierungsgesetz (BilMoG) verwirklichte der Gesetzgeber eine der umfassendsten Reformen des deutschen Handelsbilanzrechts innerhalb der letzten 20 Jahre. Unverkennbares und klar formuliertes Ziel der Reform war es, ,das bewährte HGBBilanzrecht zu einer dauerhaften und im Verhältnis zu den internationalen Rechnungslegungsstandards vollwertigen, aber kostengünstigeren und einfacheren Alternative weiterzuentwickeln, ohne die Eckpunkte des HGB-Bilanzrechts (...) und das bisherige System der Grundsätze ordnungsmäßiger Buchführung aufzugeben“. Gleichzeitig war es wesentliches Anliegen des Gesetzgebers, den Anforderungen des Kapitalmarktes nach informationsorientierteren Jahresabschlüssen gerecht zu werden und daher das Informationsniveau des handelsrechtlichen Jahresabschlusses im Rahmen der Modernisierung anzuheben. Zahlreiche handelsrechtliche Ansatz- und Bewertungsvorschriften wurden zu diesen Zwecken konzeptionell verändert und den auf europäischer und internationaler Ebene dominierenden informationsorientierten IFRS angenähert.

Während insbesondere die reformierten handelsrechtlichen Ansatz- und Bewertungsvorschriften auf Einzelabschlussebene vor dem Hintergrund der Informationsaufwertung detailliert analysiert und kritisch illustriert wurden, fand die Veränderung einer modifizierten Norm im Schrifttum nur vergleichsweise geringe Beachtung: Die Neukonzeption der handelsrechtlichen Forderung nach materieller Stetigkeit. Erstmals durch das Bilanzrichtlinien-Gesetz (BiRiLiG) im Jahre 1985 als Sollvorschrift partiell für die Bewertung kodifiziert, wurde diese im Zuge des BilMoG zum einen durch eine Wortlautveränderung des $\S 252$ Abs. 1 Nr. 6 HGB für Bewertungsmethoden in eine verpflichtende Vorschrift transformiert. Zum anderen wurde die Bewertungsmethodenstetigkeit um ein Gebot der Ansatzmethodenstetigkeit in § 246 Abs. 3 HGB erweitert. Welchen Rang und welchen Geltungsbereich dieser nunmehr totalkodifizierte, für die Bilanzierungspraxis bedeutsame Grundsatz im modernisierten HGB einnimmt, ist bisweilen ungeklärt. Eine erneute wissenschaftliche Auseinandersetzung mit diesem traditionellen und bislang nur unzureichend erschlossenen GoB ist daher unbestreitbar von Notwendigkeit.

Die vorliegende Arbeit, welche von der Wirtschafts- und Sozialwissenschaftlichen Fakultät der Universität zu Köln als Dissertation angenommen wurde, widmet sich dieser hochaktuellen Aufgabe. Sie wendet sich hierbei zunächst der Historie und der partiell für die Bewertung kodifizierten Stetigkeit zu, um im Anschluss jene Auswirkungen aufzuzeigen und umfassend zu analysieren, die sich aus der Totalkodifikation im HGB ergeben. Die Untersuchung beschränkt sich jedoch nicht nur auf das Handelsbilanzrecht, sondern setzt sich darüber hinaus kritisch mit der Anwendung sowie der Reichweite der materiellen Stetigkeit im Steuerbilanzrecht auseinander. Vor dem Hintergrund der vorgenommen Neujustierung der materiellen Maßgeblichkeit und des eingeführten steuerlichen Wahlrechtsvorbehaltes 
erhält sie damit einen umfassenden Charakter und kann bisher bestehende Forschungslücken schließen.

Die Dissertation von Herrn Wengerofsky zeichnet sich durch eine kritische und umfassende Auswertung der Literatur, der Gesetze und der Rechtsprechung sowie durch eine intensive wissenschaftliche und kritische Auseinandersetzung mit dem Komplex der materiellen Stetigkeit aus. Sie ist strukturell klar gefasst und enthält eine stets nachvollziehbare Gedankenführung. Insgesamt weist die Untersuchung einen interessanten Forschungsbeitrag auf, der das Bewusstsein dafür fördert, dass Grundsätze ordnungsmäßiger Buchführung einer stetigen Fortentwicklung bedürfen. Wissenschaftlern und Praktikern kann sie gleichermaßen ausdrücklich empfohlen werden. 


\section{Vorwort}

Die vorliegende Arbeit wurde von der Wirtschafts- und Sozialwissenschaftlichen Fakultät der Universität zu Köln im Wintersemester 2013/14 als Dissertation angenommen. Während ihrer Anfertigung habe ich die Unterstützung einer Reihe von Personen erhalten, ohne die diese Arbeit nicht in der vorliegenden Konstitution zustande gekommen wäre. Ihnen gebührt an dieser Stelle mein aufrichtiger Dank.

Mein herzlicher Dank gilt meinem akademischen Lehrer und Doktorvater Herrn Univ.-Prof. Dr. Dr. h.c. Norbert Herzig, unter dessen Betreuung ich dieses Projekt realisieren konnte. Insbesondere für das entgegengebrachte Vertrauen, die hilfreichen Anregungen sowie die stets eingeräumten Freiräume bin ich ihm gegenüber allen anderen voran zu umfassendem Dank verpflichtet. Herrn Prof. Dr. Hartmann-Wendels danke ich für die Übernahme des Zweitgutachtens und dessen zügige Erstellung. Zudem gilt mein Dank Herrn Prof. Dr. Michael Overesch für die Leitung der Prüfungskommission.

Danken möchte ich ferner allen ehemaligen und noch aktiven Doktoranden von Herrn Univ.-Prof. Dr. Dr. h.c. Norbert Herzig für die konstruktiven Diskussionen und den gemeinsamen Gedankenaustausch.

Für die Unterstützung und den Rückhalt durch meine Freunde, die in ihrem privaten Bereich von dieser Arbeit betroffen waren und mich stets positiv begleitet haben, möchte ich mich umfassend bedanken. Insbesondere Herr Philipp Pohlmann B.A., Herr Dr. Christoph Pelger sowie Herr Jun.-Prof. Dr. Duc Hung Tran, die mich während der letzten Jahre nicht nur durch ihre Freundschaft, sondern darüber hinaus durch viele konstruktive Gespräche unterstuitzt haben, sollen an dieser Stelle erwähnt sein. Bei Frau Dipl.-Bibl. Anne Scharrenberg bedanke ich mich zutiefst für ihre wertvollen Anregungen und die Zeit, die sie sich dafür genommen hat.

Nicht zuletzt durch meine Eltern Waltraud und Hugo konnte dieses Projekt erst gelingen. Ihnen gebührt umfassender Dank für die vielfältige Unterstützung während meiner Studienund Promotionszeit. 


\section{INHALTSVERZEICHNIS}

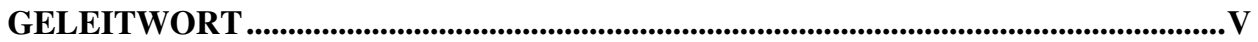

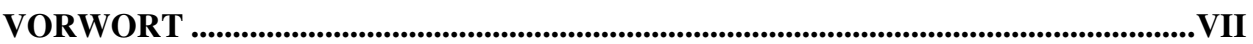

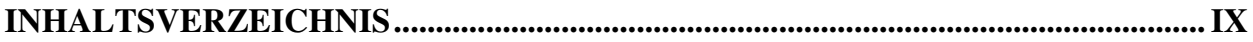

ABKÜRZUNGSVERZEICHNIS.......................................................................................XV

ABBILDUNGSVERZEICHNIS ........................................................................................XXV

TEIL 1: GRUNDLAGEN DER UNTERSUCHUNG....................................................1

Kapitel 1: $\quad$ Einführung.......................................................................................................................1

A. Relevanz des Untersuchungsgegenstandes........................................................ 1

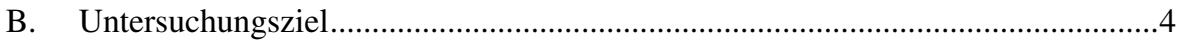

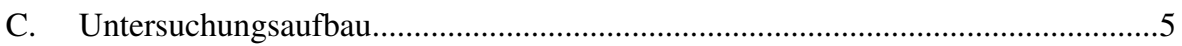

Kapitel 2: Handelsrechtliche Jahresabschlusszwecke...............................................6

A. Problematik und Methodik der Zweckbestimmung ............................................6

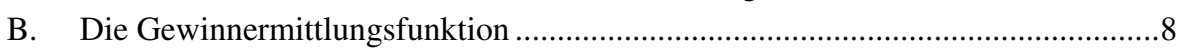

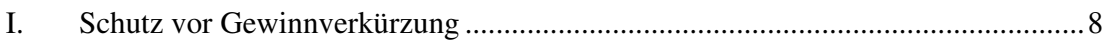

II. Schutz vor überhöhten Ausschüttungen ............................................................. 9

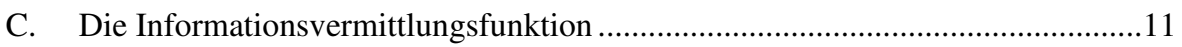

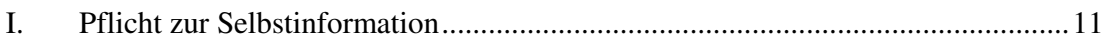

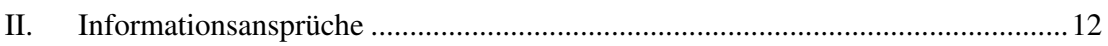

D. Zweckpluralismus der handelsrechtlichen Bilanzierung ...................................14

E. Der Rahmengrundsatz der Objektivierung als Instrument zur Erfüllung

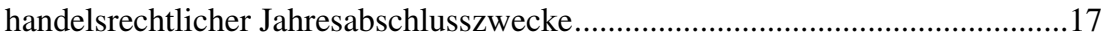

I. Ermessensbeschränkungen durch Objektivierungsrestriktionen ...........................17

II. Vergleichbarkeitsgewährleistung durch Objektivierungsrestriktionen ..................20

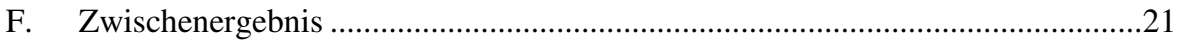

TEIL 2: DER MATERIELLE STETIGKEITSGRUNDSATZ IM HANDELSBILANZRECHT.

Kapitel 1: Der materielle Stetigkeitsgrundsatz bis zur Verabschiedung des Bilanzrechtsmodernisierungsgesetzes (BilMoG).....................................23

A. Historie des handelsrechtlichen Stetigkeitsgrundsatzes .....................................23

B. Inhaltsbestimmung des handelsrechtlich kodifizierten Stetigkeitsgrundsatzes....25

I. Der Wille des historischen Gesetzgebers als Ausgangsbasis für die Bestimmung des heutigen Norminhaltes 
II. Die europarechtlichen Vorgaben zur materiellen Stetigkeit. .26

1. Verpflichtung zur richtlinienkonformen Auslegung nationalen Rechts ...........26

2. Der Stetigkeitsgrundsatz nach der 4. EG-Richtlinie ............................................28

a. Zum Wortlaut des Art. 31 Abs. 1 Buchst. b der 4. EG-Richtlinie ......................28

(1) Stetigkeit in der Anwendung von ,Bewertungsmethoden“.............................. 28

(2) Die Soll-Formulierung des Art. 31 Abs. 1 Buchst. b der 4. EG-Richtlinie....... 29

b. Zur Zielkonzeption der 4. EG-Richtlinie ........................................................... 31

c. Zum Sinn und Zweck des Art. 31 Abs. 1 Buchst. b der 4. EG-Richtlinie .........33

d. Folgerungen für den materiellen Gehalt der Stetigkeitsforderung des Art. 31

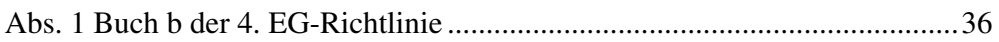

3. Der Stetigkeitsgrundsatz nach der neuen EU-Bilanzrichtlinie 2013/34/EU .....38

a. Zur Zielkonzeption der neuen EU-Bilanzrichtlinie 2013/34/EU .......................38

b. Die wesentlichen Neuerungen im Bereich der allgemeinen Ansatz- und

Bewertungsgrundsätze .

c. Implikationen für die europarechtlichen Vorgaben zur materiellen Stetigkeit.40

III. Der Begriff der „Bewertungsmethode“

1. Inhaltsbestimmung der „Bewertungsmethode“ i.S.d. § 252 Abs. 1

Nr. 6 HGB .

2. Subsumption der Ansatzmethoden unter die Bewertungsmethoden i.S.d.

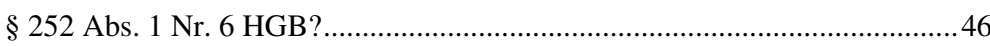

IV. Sachliche Reichweite des materiellen Stetigkeitsgrundsatzes................................50

1. Bezugsobjekte des Grundsatzes der Bewertungsstetigkeit ................................50

a. Dieselben Vermögensgegenstände und Schulden ...........................................51

b. Gleiche und gleichartige Vermögensgegenstände und Schulden ......................52

(1) Der Grundsatz der Bewertungseinheitlichkeit als integraler Bestandteil der Bewertungsstetigkeit

(2) Der Grundsatz der Bewertungseinheitlichkeit und sein Verhältnis zum Grundsatz der Einzelbewertung ..................................................................... 54

(3) Bezugspunkt für die Beurteilung der Gleichartigkeit von

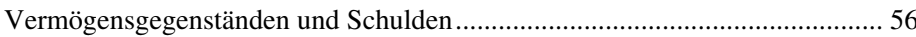

(a) Die Art- und Funktionsgleichheit als unsicherer Bezugspunkt ................................56

(b) Der normative Gesetzeszweck als sicherer Bezugspunkt.........................................59

c. Verschiedene Vermögensgegenstände und Schulden.........................................60

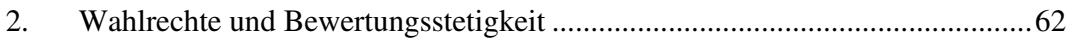

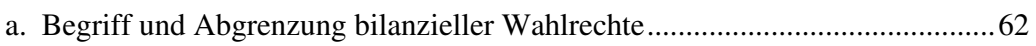

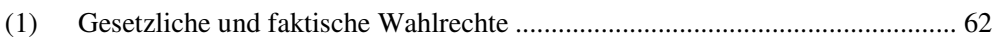

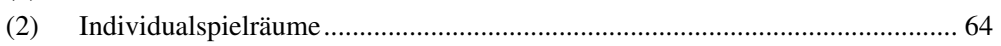

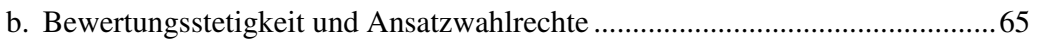

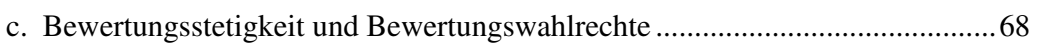

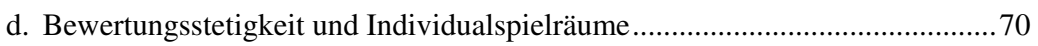

V. Zeitliche Reichweite des Stetigkeitsgrundsatzes ................................................... 71

1. Bezugnahme auf den ,,vorhergehenden Jahresabschluss“ in $\S 252$ Abs. 1 Nr. 6

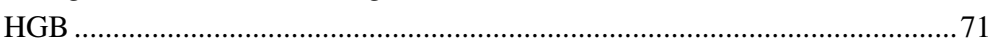

2. Auswirkungen der zeitlichen Reichweite des $§ 252$ Abs. 1 Nr. 6 HGB auf den materiellen Geltungsbereich der Bewertungsstetigkeit 
VI. Verpflichtungscharakter der Bewertungsstetigkeit gem. § 252 Abs. 1 Nr. 6 HGB a.F

1. Die Soll-Formulierung der Bewertungsstetigkeit gem. $§ 252$ Abs. 1 Nr. 6 HGB a.F. 76

a. Von der Muss-Formulierung zur Soll-Formulierung......................................... 76

b. Entstehungsgeschichte des $§ 252$ Abs. 1 Nr. 6 HGB a.F. im Rahmen des

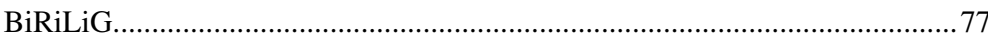

(1) Formale Gründe für die Wortlautveränderung? ............................................ 79

(2) Gesetzessystematische Interpretation der Soll-Formulierung ........................... 80

2. Auswirkungen auf den Verpflichtungscharakter ............................................. 81

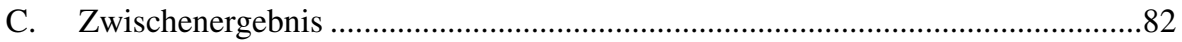

\section{Kapitel 2: Der materielle Stetigkeitsgrundsatz im HGB nach Verabschiedung des}

BilMoG 84

A. Totalkodifikation des materiellen Stetigkeitsgrundsatzes ...................................8

I. Kodifizierung eines Stetigkeitsgebotes für den Bilanzansatz..................................84

II. Wortlautänderung der kodifizierten Bewertungsstetigkeit ..................................... 84

B. Reichweite des handelsrechtlichen Stetigkeitsgrundsatzes im HGB nach

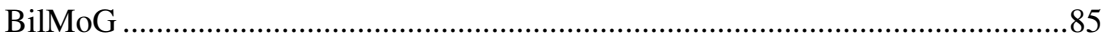

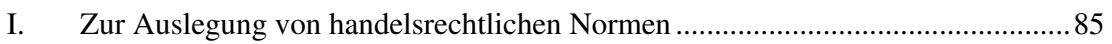

II. Implikationen für die Auslegung des Stetigkeitsgrundsatzes nach BilMoG ..........87

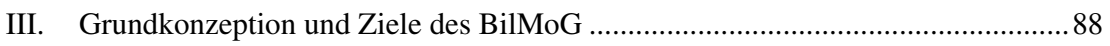

IV. Wesentliche Neuregelungen im Einzelabschluss vor dem Hintergrund der

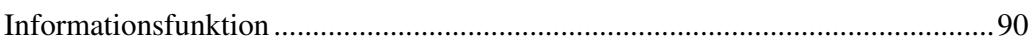

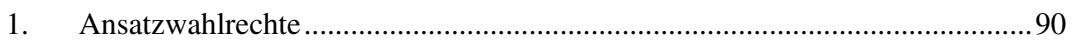

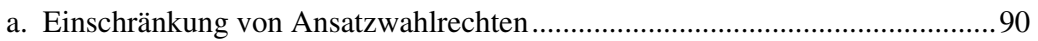

(1) Streichung von Ansatzwahlrechten ............................................................... 90

(2) Umwandlung des Ansatzwahlrechtes für den derivativen Geschäfts- oder Firmenwert in eine Ansatzpflicht .................................................................... 92

(3) Neukodifikation eines Ansatzwahlrechtes für selbsterstellte Immaterialgüter des

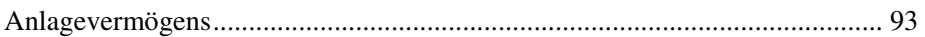

b. Beibehaltung von Ansatzwahlrechten.............................................................96

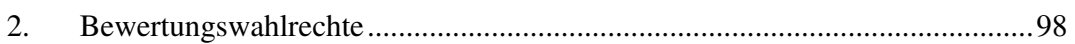

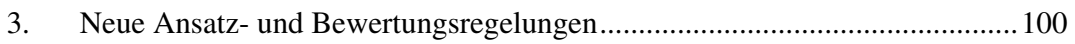

4. Die außerbilanzielle Ausschüttungssperre des § 268 Abs. 8 HGB als ergänzendes Schutzinstrument für die bilanzielle Kapitalerhaltung. 102

V. Konsequenzen für die Informationsvermittlungsfunktion des handelsrechtlichen

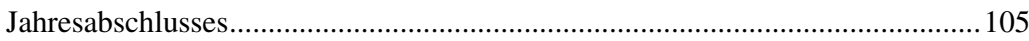

VI. Auswirkungen der veränderten Informationsvermittlung auf das

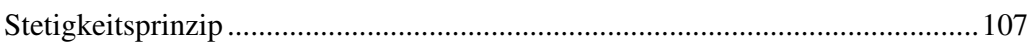

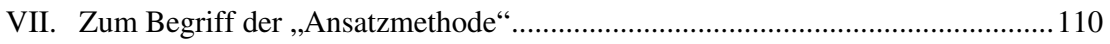

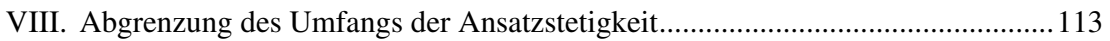

1. Sachliche Reichweite der Ansatzstetigkeit .....................................................113

a. Bezugsobjekte des Grundsatzes der Ansatzstetigkeit .................................... 113 


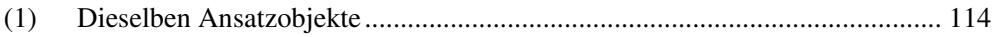

(2) Gleiche und gleichartige Ansatzobjekte ........................................................ 114

(a) Der Grundsatz der Ansatzeinheitlichkeit als integraler Bestandteil der Ansatzstetigkeit ....................................................................................... 114

(b) Bezugspunkt für die Beurteilung der Gleichartigkeit der Ansatzobjekte ............... 116

(c) Implikationen aus der Zugrundelegung des Telos der Norm als Bezugspunkt der

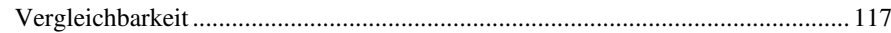

(3) Verschiedene Ansatzobjekte ................................................................. 120

2. Zeitliche Reichweite der Ansatzstetigkeit ........................................ 121

a. Bezugnahme auf den ,,vorhergehenden Jahresabschluss“ in $§ 246$ Abs. 3 Satz 1 HGB

b. Auswirkungen der zeitlichen Reichweite des $\S 246$ Abs. 3 Satz 1 HGB auf den materiellen Geltungsbereich der Ansatzstetigkeit

IX. Abweichungen von den handelsgesetzlichen Stetigkeitsprinzipien ................... 125

1. Kategorisierung der Abweichungsmöglichkeiten...................................... 125

a. Abweichungen aufgrund gesetzlicher Regelungen....................................... 126

(1) Zwingende Abweichungen aufgrund gesetzlicher Vorschriften .................... 126

(2) Zulässige Abweichungen aufgrund gesetzlicher Vorschriften...................... 128

b. Abweichungen in begründeten Ausnahmefällen ( $\$ 246$ Abs. 3 Satz 2, 252

Abs. 2 HGB)

2. Folgen einer Abweichung von den handelsgesetzlichen

Stetigkeitsgrundsätzen

3. Notwendigkeit einer rechtformspezifischen Unterscheidung der begründeten Ausnahmefälle i.S.d. §§ 246 Abs. 3 Satz 2, 252 Abs. 2 HGB? .

C. Zwischenergebnis 140

TEIL 3: DIE MATERIELLE STETIGKEIT IM STEUERBILANZRECHT 143

Kapitel 1: Die Ableitung einer materiellen Stetigkeitsforderung für die Steuerbilanz aus dem handelsgesetzlich kodifizierten GoB.

A. Kein originärer Stetigkeitsgrundsatz im Steuerbilanzrecht 143

B. Der Grundsatz der Maßgeblichkeit handelsrechtlicher GoB 144

I. Entwicklung des Verhältnisses von Handels- und Steuerbilanz .144

II. Neujustierung des Maßgeblichkeitsgrundsatzes im Rahmen des BilMoG 145

III. Reichweite des neugefassten materiellen Maßgeblichkeitsgrundsatzes und des steuerlichen Wahlrechtsvorbehalts 146

1. Materielle Maßgeblichkeit und Bilanzansatz..............................................146

a. Grundsatz der Ansatzmaßgeblichkeit ........................................................ 146

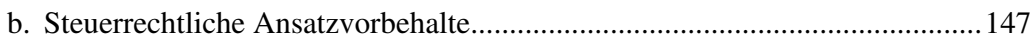

2. Materielle Maßgeblichkeit und Bewertung .................................................. 148

a. Grundsatz der Bewertungsmaßgeblichkeit .................................................. 148

b. Steuerrechtliche Bewertungsvorbehalte. 150

3. Ausweis nach den Grundsätzen ordnungsmäßiger Buchführung ( 5 Abs. 1 Satz 1, 1. Halbsatz EStG)

4. Steuerlicher Wahlrechtsvorbehalt gem. $§ 5$ Abs. 1 Satz 1, 2. Halbsatz EStG 155 


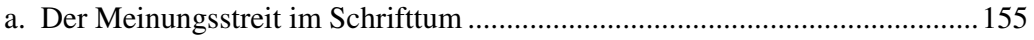

b. Die Grundwertungen der Finanzverwaltung............................................. 157

(1) Uneingeschränkte Autonomie steuergesetzlicher Wahlrechte ....................... 157

(2) Eingeschränkte Autonomie von Richtlinien-Wahlrechten ............................ 159

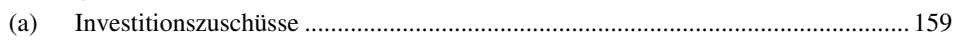

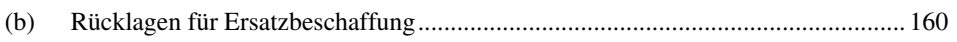

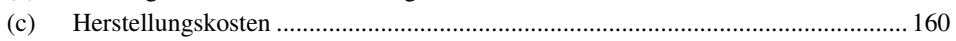

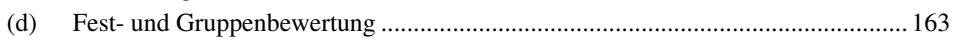

IV. Konsequenzen für die Maßgeblichkeit der materiellen handelsgesetzlichen

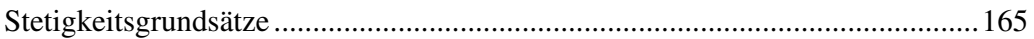

1. Handelsrechtliche Ansatzstetigkeit im Rahmen materieller

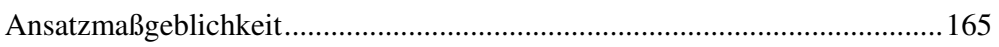

2. Handelsrechtliche Bewertungsstetigkeit im Rahmen materieller

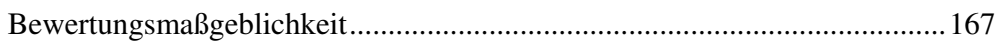

3. Keine Ansatz- und Bewertungsstetigkeit im Rahmen autonom ausgeübter

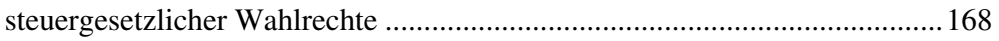

4. Keine Ansatz- und Bewertungsstetigkeit im Rahmen autonom ausgeübter Richtlinien-Wahlrechte? ...................................................................... 170

5. Ansatz- und Bewertungsstetigkeit im Rahmen von Ergänzungs- und Sonderbilanzen von Mitunternehmern.

C. Einschränkung der materiellen handelsgesetzlichen Stetigkeitsgrundsätze durch steuerrechtliche Leitprinzipien?..................................................................175

I. Handelsgesetzliche Stetigkeitsgrundsätze und Leistungsfähigkeitsprinzip.........177

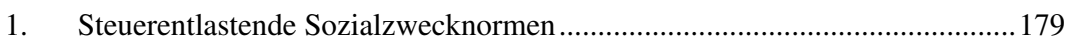

2. Vereinfachungszwecknormen ................................................................. 180

3. Folge: Doppelte Abschottung der subventionellen Steuervergünstigungen und Vereinfachungszwecknormen von den handelsgesetzlichen

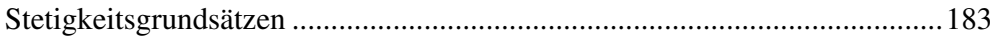

II. Handelsgesetzliche Stetigkeitsgrundsätze und Jahressteuerprinzip ..................... 185

\section{Kapitel 2: Die Ableitung einer materiellen Stetigkeitsforderung aus dem} steuerrechtlichen Grundsatz der Willkürfreiheit ..................................188

A. Die Manifestation der Stetigkeit in der steuerlichen Judikatur ..........................189

I. Konturierung der Bezeichnungen von Stetigkeitsforderungen in der höchstrichterlichen Rechtsprechung.

II. Willkürfreiheit als Rechtfertigungsgrundlage von materiellen

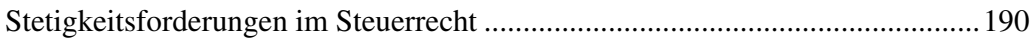

III. Inhalt und Reichweite des Grundsatzes der Willkürfreiheit................................ 191

IV. Exkurs: Abgrenzung Willkürfreiheit und Willkürverbot ..................................... 193

V. Höchstrichterliche Steuerrechtsprechung zum Grundsatz der Willkürfreiheit.... 195

1. Willkürfreie, stetige Erfolgsermittlung im Bereich der Bewertung................. 195

2. Willkürfreie, stetige Erfolgsermittlung im Bereich des Ansatzes ...................197

3. Begründungen der Willkürfreiheit in der steuerlichen Rechtsprechung......... 199

a. Element der Grundsätze ordnungsmäßiger Buchführung ...............................200 
b. Verstoß gegen den Grundsatz von Treu und Glauben .......................................201

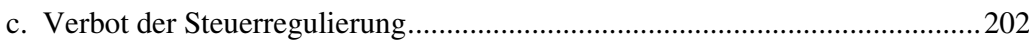

B. Folgen für materielle Stetigkeitsforderungen bei der Ausübung von originär steuerlichen Wahlrechten und Rechnungslegungsspielräumen

I. Veränderte Deduktionsbasis als Ursache für einen von der handelsgesetzlichen

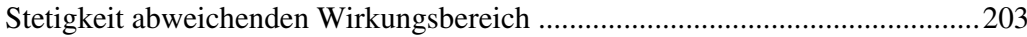

II. Erfassung von Freiheiten dem Grunde und der Höhe nach..................................204

III. Begrenzung des Wirkungsbereiches auf das einzelne Ansatz- bzw.

Bewertungsobjek

RECHTSQUELLENVERZEICHNIS ........................................................................281

RECHTSPRECHUNGSVERZEICHNIS .............................................................................286

VERZEICHNIS DER RICHTLINIEN UND VERWALTUNGSANWEISUNGEN...293 


\section{ABKÜRZUNGSVERZEICHNIS}

A

a.A.

anderer Ansicht

a.F.

alte Fassung

AB1. EG

Amtsblatt der Europäischen Gemeinschaften (bis Januar 2003)

AB1. EU

Amtsblatt der Europäischen Union (seit Februar 2003)

Abs.

Absatz

Abt.

Abteilung

AEUV

Vertrag über die Arbeitsweise der Europäischen Union

AfA

Absetzung für Abnutzung

AG

Die Aktiengesellschaft (Zeitschrift)

AktG

Aktiengesetz

Anm.

Anmerkung(en)

AO

Abgabenordnung

Art.

Artikel

ASC

Accounting Standards Codification

Az.

Aktenzeichen

B

BB

Betriebs-Berater (Zeitschrift)

BBK

Zeitschrift für Buchführung, Bilanzierung, Kostenrechnung (Zeitschrift)

BBP

Betriebswirtschaft im Blickpunkt (Zeitschrift)

BC

Bilanzbuchhalter und Controller (Zeitschrift)

Beck Bil-Komm

Beck'scher Bilanz-Kommentar

Beck HdGmbH

Beck'sches Handbuch der GmbH

Beil.

Beilage

ber.

berichtigt

BFA

Bankenfachausschuss

$\mathrm{BFH} / \mathrm{NV}$

Sammlung amtlich nicht veröffentlichter Entscheidungen des BFH (Zeitschrift) 
BFHE

$\mathrm{BFuP}$

BGB

BGB1.

BGH

BilMoG

BilMoG-RefE

BilMoG-RegE

BilReG

BiRiLiG

BMF

BMJ

BoHR

BR

BR-Drucks.

bspw.

BStB1.

BT

BT-Drucks.

Buchst.

BuW

BVerfG

BVerfGE

bzgl.

bzw.

C

CDU

CSU
Sammlungen der Entscheidungen und Gutachten des

$\mathrm{BFH}$

Betriebswirtschaftliche Forschung und Praxis (Zeitschrift)

Bürgerliches Gesetzbuch

Bundesgesetzblatt

Bundesgerichtshof

Bilanzrechtsmodernisierungsgesetz

Bilanzrechtsmodernisierungsgesetz-Referentenentwurf

Bilanzrechtsmodernisierungsgesetz-Regierungsentwurf

Bilanzrechtsreformgesetz

Bilanzrichtlinien-Gesetz

Bundesministerium der Finanzen

Bundesministerium der Justiz

Bonner Handbuch Rechnungslegung

Bundesrat

Drucksache des Bundesrats

beispielsweise

Bundessteuerblatt

Bundestag

Drucksache des Bundestags

Buchstabe

Betrieb und Wirtschaft (Zeitschrift)

Bundesverfassungsgericht

Entscheidungen des Bundesverfassungsgerichts

bezüglich

beziehungsweise

Christlich Demokratische Union Deutschlands

Christlich-Soziale Union in Bayern e.V. 
D

d.h.

das heißt

DB

Der Betrieb (Zeitschrift)

DBW

Die Betriebswirtschaft (Zeitschrift)

Diss.

Dissertation

DK

Der Konzern (Zeitschrift)

$\mathrm{DM}$

Deutsche Mark

Dr.

Doktor

DRS

Deutscher Rechnungslegungs Standard

DRSC

Deutsches Rechnungslegungs Standards Committee

Drucks.

Drucksache

DSR

Deutscher Standardisierungsrat

DStJG

Veröffentlichungen der Deutschen Steuerjuristischen Gesellschaft e.V. (Zeitschrift)

DStR

Deutsches Steuerrecht (Zeitschrift)

DStZ

Deutsche Steuer-Zeitung (Zeitschrift)

DVFA

Deutsche Vereinigung für Finanzanalyse und Asset Management

$\mathbf{E}$

e.V.

eingetragener Verein

EFG

Entscheidungen der Finanzgerichte

EG

Europäische Gemeinschaft(en)

EGAO

Einführungsgesetz zur Abgabenordnung

EGV

Vertrag zur Gründung der Europäischen Gemeinschaft

EGHGB

Einführungsgesetz zum Handelsgesetzbuch

EStÄR

Einkommensteuer-Änderungsrichtlinie

EStB

Der Ertragsteuer-Berater (Zeitschrift)

EStG

Einkommensteuergesetz

EStG-E

Einkommensteuergesetz-Entwurf

EStR

Einkommensteuer-Richtlinien

EStR-E

Einkommensteuer-Richtlinien-Entwurf

et al.

et alii/ und andere 
$\mathrm{EuGH}$

EuR

EU

EUV

EWG

F

f.

FDP

ff.

FG

Fifo

Fn.

FR

G

GAAP

gem.

GG

ggf.

GK-HGB

$\mathrm{GmbH}$

GmbHG

GmbHR

$\mathrm{GmbH}-\mathrm{StB}$

GoB

grds.

GrS

$\mathrm{GuV}$

GWG
Europäischer Gerichtshof

Europarecht (Zeitschrift)

Europäische Union

Vertrag über die Europäische Union

Europäische Wirtschaftsgemeinschaft

folgende

Freie Demokratische Partei

fortfolgende

Finanzgericht

First in - first out

Fußnote

Finanz-Rundschau (Zeitschrift)

Generally Accepted Accounting Principles

gemäß

Grundgesetz

gegebenenfalls

Gemeinschaftskommentar zum HGB

Gesellschaft mit beschränkter Haftung

Gesetz betreffend die Gesellschaften mit beschränkter Haftung

GmbH-Rundschau (Zeitschrift)

GmbH-Steuerberater (Zeitschrift)

Grundsätze ordnungsmäßiger Buchführung

grundsätzlich

Großer Senat

Gewinn- und Verlustrechnung

Geringwertiges Wirtschaftsgut

xviii 
$\mathbf{H}$

Habil.

Habilitationsschrift

HdB

Handbuch der Bilanzierung

HdBetr.

Handwörterbuch der Betriebswirtschaft

HdR

Handbuch der Rechnungslegung

HdJ

Handbuch des Jahresabschlusses

HFA

Hauptfachausschuss

HFR

Höchstrichterliche Finanzrechtsprechung (Zeitschrift)

HGB

Handelsgesetzbuch

HGB-E

Handelsgesetzbuch-Entwurf

$\mathrm{H} / \mathrm{H} / \mathrm{R}$

Hermann/Heuer/Raupach (Kommentar)

Hifo

Highest in - first out

HK-HGB

Heidelberger Kommentar zum HGB

HLBS

Hauptverband der landwirtschaftlichen Buchstellen und Sachverständigen e.V.

Hrsg.

Herausgeber

HURB

HwdB

Handwörterbuch unbestimmter Rechtsbegriffe im Bilanzrecht des HGB

HWRP

Handwörterbuch der Betriebswirtschaft

HWRW

Handbuch der Rechnungslegung und Prüfung

Handwörterbuch des Rechnungswesens

\section{I}

i.d.R.

in der Regel

i.e.S.

im engeren Sinne

i.S.d.

im Sinne des

i.S.v.

im Sinne von

IAS

International Accounting Standards

IASB

International Accounting Standards Board

IDW

Institut der Wirtschaftsprüfer in Deutschland e.V.

IDW-FN

Fachnachrichten des Instituts der Wirtschaftsprüfer in Deutschland e.V. (Zeitschrift) 
IFRS

IFSt

INF

insbes.

IT

J

JbFfStR

$\mathrm{Jg}$.

$\mathrm{JZ}$

K

$\mathrm{K} / \mathrm{S} / \mathrm{M}$

Kap.

$\mathrm{KiFo}$

Kilo

KMU

Komm.

KoR

KölnKomm-ReLe-Recht

KÖSDI

L

$\mathrm{L} / \mathrm{B} / \mathrm{P}$

$\mathrm{LdR}$

LG

Lifo

LoFo

LSt
International Financial Reporting Standards

Institut Finanzen und Steuern e.V.

Die Information über Steuer und Wirtschaft (Zeitschrift)

insbesondere

Informationstechnik

Jahrbuch der Fachanwälte für Steuerrecht

Jahrgang

Juristen-Zeitung (Zeitschrift)

Kirchhof/Söhn/Mellinghoff (Kommentar)

Kapitel

Konzern in - First out

Konzern in - last out

Kleine und mittlere Unternehmen

Kommentar

Kapitalmarktorientierte Rechnungslegung (Zeitschrift)

Kölner Kommentar zum Rechnungslegungsrecht

Kölner Steuerdialog (Zeitschrift)

Littmann/Bitz/Pust (Kommentar)

Lexikon des Rechnungswesens

Landgericht

Last in - first out

Lowest in - First out

Lohnsteuer 
m.w.N.

MAH

MEZ

MicroBilG

MünchKomm AktG

$\mathbf{N}$

n.F.

NB

NJW

NJW-RR

No.

Nr.

NVwZ

NWB

O

OFD

OLG

$\mathbf{P}$

p.a.

PiR

Prof.

PS

PublG

$\mathbf{R}$

$\mathrm{R}$

RefE

RegE mit weiteren Nachweisen

Münchener Anwaltshandbuch

Mitteleuropäische Zeit

Kleinstkapitalgesellschaften-Bilanzrechtsänderungsgesetz

Münchener Kommentar zum Aktiengesetz

neue Fassung

Neue Betriebswirtschaft (Zeitschrift)

Neue Juristische Wochenschrift (Zeitschrift)

NJW-Rechtsprechungs-Report Zivilrecht (Zeitschrift)

Number

Nummer

Neue Zeitschrift für Verwaltungsrecht (Zeitschrift)

Neue Wirtschafts-Briefe

Oberfinanzdirektion

Oberlandesgericht

per annum

Praxis der internationalen Rechnungslegung (Zeitschrift)

Professor

Prüfungsstandard

Publizitätsgesetz

Richtlinie

Referentenentwurf

Regierungsentwurf 
REStG

RFH

RFHE

RGB1.

Rs.

RS

RStB1.

Rz.

S

S.

Schr.

Schrb.

SFAS

Slg.

sog.

Sp.

SPD

$\mathrm{S}: \mathrm{R}$

$\mathrm{StB}$

Stbg

StbJb

StBp

StEuglG

SteuK

StuB

StuW

$\mathrm{StVj}$

StWa
Reichseinkommensteuergesetz

Reichsfinanzhof

Amtliche Sammlung der Entscheidungen des Reichsfinanzhofs

Reichsgesetzblatt

Rechtssache

Stellungnahme zur Rechnungslegung

Reichssteuerblatt

Randziffer

Seite

Schrift

Schreiben

Statement of Financial Accounting Standards

Sammlung der Rechtsprechung des Gerichtshofs EuGH und des

Gerichts Erster Instanz

so genannte

Spalte

Sozialdemokratische Partei Deutschlands

Status: Recht (Zeitschrift)

Der Steuerberater (Zeitschrift)

Die Steuerberatung (Zeitschrift)

Steuerberater-Jahrbuch

Die steuerliche Betriebsprüfung (Zeitschrift)

Steuer-Euroglättungsgesetz

Steuerrecht kurzgefaßt (Zeitschrift)

Steuern und Bilanzen (Zeitschrift)

Steuer und Wirtschaft (Zeitschrift)

Steuerliche Vierteljahresschrift (Zeitschrift)

Die Steuerwarte (Zeitschrift) 
T

Techn. Univ.

$\mathbf{U}$

u.

u.a.

u.a.m.

u.d.T.

Ubg

Univ.

US, USA

US-GAAP

USt

usw.

V

V.

v.a.

vergl.

VFE-Lage

vgl.

VO

VW

W

WiSt

WM

WoBauFG

WPg
Technische Universität

und

und andere/ unter anderem

und andere(s) mehr

unter dem Titel

Die Unternehmensbesteuerung (Zeitschrift)

Universität

United States of America

United States Generally Accepted Accounting Principles

Umsatzsteuer

und so weiter

von, vom

vor allem

vergleichbar

Vermögens-, Finanz- und Ertragslage

vergleiche

Verordnung

Versorgungs Wirtschaft (Zeitschrift)

Wirtschaftswissenschaftliches Studium (Zeitschrift)

Wertpapier-Mitteilungen (Zeitschrift)

Wohnbauförderungsgesetz

Die Wirtschaftsprüfung (Zeitschrift) 
z.B.

ZCG

ZEW

ZfB

$\mathrm{ZfbF}$

ZfgK

ZfhF

ZGR

ZHR

Ziff.

ZPlan

$\mathrm{ZStF}$

ZSteu

zugl. zum Beispiel

Zeitschrift für Corporate Governance

Zentrum für Europäische Wirtschaftsforschung GmbH

Zeitschrift für Betriebswirtschaft

Schmalenbachs Zeitschrift für betriebswirtschaftliche Forschung

Zeitschrift für das gesamte Kreditwesen

Zeitschrift für handelswissenschaftliche Forschung

Zeitschrift für Unternehmens- und Gesellschaftsrecht

Zeitschrift für das gesamte Handels- und Wirtschaftsrecht

Ziffer

Zeitschrift für Planung \& Unternehmensteuerung

Zeitgemäße Steuer- und Finanzfragen (Zeitschrift)

Zeitschrift für Steuern \& Recht

zugleich 


\section{ABBILDUNGSVERZEICHNIS}

Abbildung 1: Handelsrechtliche Jahresabschlusszwecke 14

Abbildung 2: Die Zielkonzeption der 4. EG-Richtlinie 33

Abbildung 3: Das materielle Stetigkeitsprinzip gem. Art. 31 Abs. 1 Buchst. b der 4. EG-Richtlinie 36

Abbildung 4: Zur Zielkonzeption der EU-Bilanzrichtlinie 2013/34/EU 39

Abbildung 5: Die Prozessstufen einer Bewertungsmethode 46

Abbildung 6: Abgrenzung echte Wahlrechte und Rechnungslegungsspielräume 65

Abbildung 7: Das materielle Stetigkeitsprinzip gem. § 252 Abs. 1 Nr. 6 HGB a.F....... 83

Abbildung 8: Ziele des BilMoG 90

Abbildung 9: Erstellungsprozess eines immateriellen Vermögensgegenstandes 96

Abbildung 10: Kategorisierung der Abweichungsmöglichkeiten 134

Abbildung 11: Der Grundsatz der materiellen Stetigkeit im modernisierten HGB 141

Abbildung 12: Der Grundsatz der Maßgeblichkeit, modifiziert entnommen aus Federmann, Bilanzierung, 12. Auflage 2010, S. 241 164

Abbildung 13: Abgrenzung von Willkürfreiheit und Willkürverbot 195 